International Mathematical Forum, Vol. 8, 2013, no. 24, 1155 - 1162

HIKARI Ltd, www.m-hikari.com

http://dx.doi.org/10.12988/imf.2013.3485

\title{
States on Subtraction Algebras
}

\author{
Sang Moon Lee \\ Department of Computer Science and Information Engineering \\ Korea National University of Transportation \\ Chungju 380-702, Korea \\ smlee@ut.ac.kr \\ Kyung Ho Kim \\ Department of Mathematics \\ Korea National University of Transportation \\ Chungju 380-702, Korea \\ ghkim@ut.ac.kr
}

Copyright (c) 2013 Sang Moon Lee and Kyung Ho Kim. This is an open access article distributed under the Creative Commons Attribution License, which permits unrestricted use, distribution, and reproduction in any medium, provided the original work is properly cited.

\begin{abstract}
In this paper, we study the Bosbach state on subtraction algebra and show that there exists a Bosbach state via $X / \theta$ where $\theta$ is a congruence relation induced by an ideal of $X$.
\end{abstract}

Mathematics Subject Classification: 06F35, 03G25, 08A30

Keywords: Subtraction algebra, Bosbach state, Ker (s), congruence class

\section{Introduction}

B. M. Schein [3] considered systems of the form $(\Phi ; \circ, \backslash)$, where $\Phi$ is a set of functions closed under the composition "O" of functions (and hence $(\Phi ; \circ)$ is a function semigroup) and the set theoretic subtraction " $"$ " (and hence $(\Phi ; \backslash)$ is a subtraction algebra in the sense of [1]. He proved that every subtraction semigroup is isomorphic to a difference semigroup of invertible functions. B. 
Zelinka [4] discussed a problem proposed by B. M. Schein concerning the structure of multiplication in a subtraction semigroup. He solved the problem for subtraction algebras of a special type, called the atomic subtraction algebras. In this paper, we study the Bosbach state on subtraction algebra and show that there exists a Bosbach state via $X / \theta$ where $\theta$ is a congruence relation induced by an ideal of $X$.

\section{Subtraction algebras}

By a subtraction algebra we mean an algebra $(X ;-)$ with a single binary operation "-" that satisfies the following identities: for any $x, y, z \in X$,

(S1) $x-(y-x)=x$;

(S2) $x-(x-y)=y-(y-x)$;

(S3) $(x-y)-z=(x-z)-y$.

The last identity permits us to omit parentheses in expressions of the form $(x-y)-z$. The subtraction determines an order relation on $X: a \leq b \Leftrightarrow$ $a-b=0$, where $0=a-a$ is an element that does not depend on the choice of $a \in X$. The ordered set $(X ; \leq)$ is a semi-Boolean algebra in the sense of [6], that is, it is a meet semilattice with zero 0 in which every interval $[0, a]$ is a Boolean algebra with respect to the induced order. Here $a \wedge b=a-(a-b)$; the complement of an element $b \in[0, a]$ is $a-b$; and if $b, c \in[0, a]$, then

$$
\begin{aligned}
b \vee c & =\left(b^{\prime} \wedge c^{\prime}\right)^{\prime}=a-((a-b) \wedge(a-c)) \\
& =a-((a-b)-((a-b)-(a-c))) .
\end{aligned}
$$

In a subtraction algebra, the following are true: for all $x, y, z \in X$,

(p1) $(x-y)-y=x-y$.

(p2) $x-0=x$ and $0-x=0$.

(p3) $x-y \leq x$.

(p4) $x-(x-y) \leq y$.

(p5) $(x-y)-(y-x)=x-y$.

(p6) $x-(x-(x-y))=x-y$.

(p7) $(x-y)-(z-y) \leq x-z$.

(p8) $x \leq y$ if and only if $x=y-w$ for some $w \in X$. 
(p9) $x \leq y$ implies $x-z \leq y-z$ and $z-y \leq z-x$ for all $z \in X$.

(p10) $x, y \leq z$ implies $x-y=x \wedge(z-y)$.

(p11) $(x \wedge y)-(x \wedge z) \leq x \wedge(y-z)$.

(p12) $(x-y)-z=(x-z)-(y-z)$.

A non-empty subset $I$ of a subtraction algebra $X$ is called a subalgebra if $x-y \in I$ for all $x, y \in I$. A mapping $f$ from a subtraction algebra $X$ to a subtraction algebra $Y$ is called a homomorphism if $f(x-y)=f(x)-f(y)$ for all $x, y \in X$. A homomorphism $f$ from a subtraction algebra $X$ to itself is called an endomorphism of $X$. If $x \leq y$ implies $f(x) \leq f(y), f$ is called an isotone map.

A nonempty subset $I$ of a subtraction algebra $X$ is called an ideal of $X$ if it satisfies the following:

(I1) $0 \in I$,

(I2) for any $x, y \in X, y \in I$ and $x-y \in I$ imply $x \in I$.

Lemma 2.1. Let $(X ;-)$ be a subtraction algebra and $I$ a nonempty subset of $X$. Then $I$ is an ideal of $X$ if and only if it satisfies the following properties:

(1) $x \in I$ and $y \leq x$ imply $y \in I$,

(2) if $x, y \in I$ and $x \vee y$ exists, then $x \vee y \in I$.

\section{$3 \quad$ States on subtraction algebras}

Definition 3.1. Let $X$ be a subtraction algebra. A Bosbach state on $X$ is a function $s: X \rightarrow[0,1]$ such that the following axioms hold:

(i) $s(0)=0$,

(ii) $s(x)+s(y-x)=s(y)+s(x-y)$ for all $x, y \in X$.

For a Bosbach state $s: X \rightarrow[0,1]$, define a set $\operatorname{Ker}(s)$ by

$$
\operatorname{Ker}(s)=\{x \in X \mid s(x)=0\} .
$$

A function $s: X \rightarrow[0,1]$ is called a trivial Bosbach state on $X$ if $s(x)=0$ for all $x \in X$. Also, the function

$$
s(x)= \begin{cases}0 & \text { if } x=0, \\ t & \text { otherwise }\end{cases}
$$

is called a constant Bosbach state for some $t \in[0,1]$. 
Example 3.2. Let $X=\{0, a, b\}$ be a set in which "-" is defined by

\begin{tabular}{c|ccc}
- & 0 & $a$ & $b$ \\
\hline 0 & 0 & 0 & 0 \\
$a$ & $a$ & 0 & $a$ \\
$b$ & $b$ & $b$ & 0
\end{tabular}

Define a function $s: X \rightarrow[0,1]$ by

$$
s(0)=0, s(a)=0.3, s(b)=0.7 \text {. }
$$

Then $s$ is a Bosbach state on $X$.

Example 3.3. Let $X=\{0, x, y, 1\}$ be a set in which "-" is defined by

\begin{tabular}{c|cccc}
- & 0 & $x$ & $y$ & 1 \\
\hline 0 & 0 & 0 & 0 & 0 \\
$x$ & $x$ & 0 & $x$ & 0 \\
$y$ & $y$ & $y$ & 0 & 0 \\
1 & 1 & $y$ & $x$ & 0
\end{tabular}

Define a function $s: X \rightarrow[0,1]$ by

$$
s(0)=0, s(x)=0.3, s(y)=0.5, s(1)=0.8 .
$$

Then $s$ is a Bosbach state on $X$.

Proposition 3.4. If $s: X \rightarrow[0,1]$ is a Bosbach state on $X, s_{a}: X \rightarrow$ $[0,1], s_{a}(x)=s(x-a)$ for every $x \in X$ is also a Bosbach state on $X$.

Proof. Let $X$ be a subtraction algebra and $a \in X$. Then we have

$$
s_{a}(0)=s(0-a)=s(0)=0 .
$$

Also, we have for all $x, y \in X$,

$$
\begin{aligned}
s_{a}(x)+s_{a}(y-x) & =s(x-a)+s((y-x)-a)) \\
& =s(x-a)+s((y-a)+(x-a)) \\
& =s(y-a)+s((x-a)+(y-a)) \\
& =s(y-a)+s((x-y)-a) \\
& =s_{a}(y)+s_{a}(x-y) .
\end{aligned}
$$

This completes the proof. 
Proposition 3.5. Let $s: X \rightarrow[0,1]$ be a Bosbach state on $X$. Then If $x \leq y$, then $s(x) \leq s(y)$ and $s(x)+s(y-x)=s(y)$.

Proof. Let $s$ be a Bosbach state on $X$ and $x \leq y$. Then $x-y=0$. Since $s(x)+s(y-x)=s(y)+s(x-y)$, we have $0 \leq s(y-x)=s(x-y)+s(y)-s(x)=$ $0+s(y)-s(x)=s(y)-s(x)$, and so $s(y) \geq s(x)$.

Proposition 3.6. Let $s: X \rightarrow[0,1]$ be a Bosbach state on $X$. Then $\operatorname{Ker}(s)$ is a subalgebra of $X$.

Proof. Let $x, y \in \operatorname{Ker}(s)$. Then $s(x)=s(y)=0$. Since $x-y \leq x$, we have $s(x-y) \leq s(x)$ by Proposition 3.5, and so $0 \leq s(x-y) \leq s(x)=0$. This implies $s(x-y)=0$, i.e., $x-y \in \operatorname{Ker}(s)$.

Proposition 3.7. Let $s: X \rightarrow[0,1]$ be a Bosbach state on $X$. Then $\operatorname{Ker}(s)$ is an ideal of $X$.

Proof. Since $s(0)=0$, we have $0 \in \operatorname{Ker}(s)$. Let $x-y \in \operatorname{Ker}(s)$ and $y \in$ $\operatorname{Ker}(s)$. So, $s(x-y)=s(y)=0$. Since $y-x \leq y$, we have $s(y-x) \leq s(y)$ by Proposition 3.5, which implies $0 \leq s(x-y) \leq s(y)=0$. Hence $s(y-x)=0$. Now, since $s(x)+s(y-x)=s(y)+s(x-y)$, we obtain $s(x)+0=0+0$. This implies $s(x)=0$, i.e., $x \in \operatorname{Ker}(s)$.

Theorem 3.8. Let $f: X \rightarrow Y$ be a homomorphism and $s: Y \rightarrow[0,1]$ be a Bosbach state on $Y$. Then there exists an unique Bosbach state $t: X \rightarrow[0,1]$ such that the following diagram is commutative (i.e., $t=s \circ f$ ).

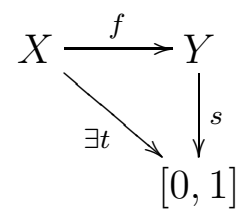

Proof. Let $t:=s \circ f$. It is clear that $t$ is well-defined. Now we show that $t$ is a Bosbach state. We know that $t(0)=(s \circ f)(0)=s(f(0))=s(0)=0$. Now, 
let $x, y \in X$. Then

$$
\begin{aligned}
t(x)+t(y-x) & =(s \circ f)(x)+(s \circ f)(y-x) \\
& =s(f(x))+s(f(y)-f(x)) \\
& =s(f(y))+s(f(x)-f(y)) \\
& =s(f(y))+s(f(x-y)) \\
& =(s \circ f)(y)+(s \circ f)(x-y) \\
& =t(y)+t(x-y) .
\end{aligned}
$$

Hence $t$ is a Bosbach state. Finally, we prove that $t$ is unique. Suppose that there exists an another Bosbach state $r: X \rightarrow[0,1]$ such that $r=s \circ f$. Then $r(x)=(s \circ f)(x)=t(x)$ for all $x \in X$. Hence we have $r=t$.

Theorem 3.9. Let $f: X \rightarrow Y$ be a bijection homomorphism and $s: X \rightarrow$ $[0,1]$ be a Bosbach state on $X$. Then there exists an unique Bosbach state $t: Y \rightarrow[0,1]$ such that $s=t \circ f$.

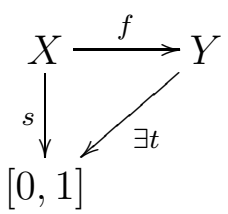

Proof. Let $y \in Y$. Since $f$ is onto, there exists $x \in X$ such that $f(x)=y$. Put $t(y)=s(x)$. Then $t(y)=t(f(x))=(t \circ f)(x)=s(x)$, and so $t \circ f=s$. Since $f$ is one to one, then $\operatorname{Ker}(f)=0$, and so $t(0)=t(f(0))=(t \circ f)(0)=s(0)=0$. Let $y_{1}, y_{2} \in Y$. Then there exists $x_{1}, x_{2} \in X$ such that $f\left(x_{1}\right)=y_{1}$ and $f\left(x_{2}\right)=y_{2}$. Then we have

$$
\begin{aligned}
t\left(y_{1}\right)+t\left(y_{2}-y_{1}\right) & =t\left(f\left(x_{1}\right)+t\left(f\left(x_{2}\right)-f\left(x_{1}\right)\right)\right. \\
& =t\left(f\left(x_{1}\right)+t\left(f\left(x_{2}-x_{1}\right)\right)\right. \\
& =(t \circ f)\left(x_{1}\right)+(t \circ f)\left(x_{2}-x_{1}\right) \\
& =s\left(x_{1}\right)+s\left(x_{2}-x_{1}\right) \\
& =s\left(x_{2}\right)+s\left(x_{1}-x_{2}\right) \\
& =(t \circ f)\left(x_{2}\right)+(t \circ f)\left(x_{1}-x_{2}\right) \\
& =\left(t \left(f\left(x_{2}\right)+t\left(f\left(x_{1}-x_{2}\right)\right)\right.\right. \\
& =t\left(f\left(x_{2}\right)\right)+t\left(f\left(x_{1}\right)-f\left(x_{2}\right)\right) \\
& =t\left(y_{2}\right)+t\left(y_{1}-y_{2}\right) .
\end{aligned}
$$

Hence $t$ is a Bosbach state. Suppose that there exists an another Bosbach state $r: X \rightarrow[0,1]$ such that $s=r \circ f$. Let $y \in Y$. Then there exists $x \in X$ 
such that $f(x)=y$. Now, $r(y)=r(f(x))=(r \circ f)(x)=s(x)$, but by definition of $t$, we get $r(y)=s(x)=t(y)$. Hence we have $r=t$.

Let $I$ be an ideal of $X$. Consider relation $\theta$ on $X$ by

$$
(x, y) \in \theta \quad \Leftrightarrow \quad x-y \in I \text { and } y-x \in I .
$$

Then $\theta$ is a congruence relation on $X$. For $x \in X$, we denote by $[x]$ the congruence class of $x$ and let

$$
X / \theta=\{[x] \mid x \in X\} .
$$

Then $X / \theta$ is a subtraction algebra where $[x]-[y]=[x-y]$ for all $x, y \in X$. Also, we denote $p_{\theta}: X \rightarrow X / \theta$ by $p_{\theta}(x)=[x]$. If $x \in I$, we have $[x]=[0]=0$. We introduce a relation " $\leq$ " on $X / \theta$ by $[x] \leq[y]$ if and only if $[x]-[y]=[0]=0$.

Proposition 3.10. Let $s: X \rightarrow[0,1]$ be a Bosbach state on $X$. Then

(i) $[x] \leq[y] \Leftrightarrow s(x-y)=0$.

(ii) $[x]=[y] \Leftrightarrow s(x-y)=s(y-x)=0$.

Proof. It is easy to prove from Proposition 3.5.

Theorem 3.11. Let $I$ be an ideal of $X$ and $s: X \rightarrow[0,1]$ be a Bosbach state on $X$. Then there exists an unique Bosbach state $t: X / \theta \rightarrow[0,1]$ such that the following diagram is commutative (i.e., $s=t \circ p_{\theta}$ ), in fact, $\theta$ is a congruence relation induced by ideal $I$.

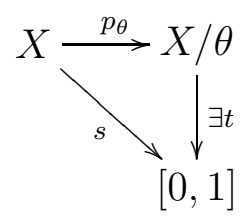

Corollary 3.12. Let $X$ be a subtraction algebra and $s: X \rightarrow[0,1]$ be a Bosbach state on $X$. Then there exists an unique Bosbach state $t: X / \theta \rightarrow[0,1]$ such that the following diagram is commutative (i.e., $s=t \circ p_{\theta}$ ), in fact, $\theta$ is a congruence relation induced by ideal $\operatorname{Ker}(s)$.

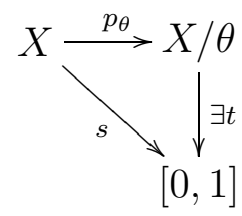




\section{References}

[1] J. C. Abbott, Sets, Lattices and Boolean Algebras, Allyn and Bacon, Boston 1969.

[2] J. C. Abbott, Algebras of implication and semi-lattices, Séminarire Dubreil (Algèbre et théorie des nombres) 20(2) (1966-1967), exp. $n^{0} 20$, 1-8.

[3] B. M. Schein, Difference Semigroups, Comm. in Algebra 20 (1992), 21532169.

[4] B. Zelinka, Subtraction Semigroups, Math. Bohemica, 120 (1995), 445447.

Received: April 21, 2013 